\title{
Current Modalities for the Assessment of Future Remnant Liver Function
}

\author{
Fadi Rassam $^{a}$ Pim B. Olthof ${ }^{a}$ Roelof J. Bennink ${ }^{b}$ Thomas M. van Gulik ${ }^{a}$ \\ ${ }^{a}$ Department of Surgery, Academic Medical Center, Amsterdam, The Netherlands; \\ ${ }^{\mathrm{b}}$ Department of Radiology and Nuclear Medicine, Academic Medical Center, Amsterdam, The Netherlands
}

\section{Keywords}

Liver function tests - Liver resection - Posthepatectomy liver failure

\section{Summary}

While imaging studies such as computed tomography or magnetic resonance imaging allow the volumetric assessment of the liver segments, only indirect information is provided concerning the quality of the liver parenchyma and its actual functional capacity. Assessment of liver function is therefore crucial in the preoperative workup of patients who require extensive liver resection and in whom portal vein embolization is considered. This review deals with the modalities currently available for the measurement of liver function. Passive liver function tests include biochemical parameters and clinical grading systems such as the Child-Pugh and MELD scores. Dynamic quantitative tests of liver function can be based on clearance capacity tests such as the indocyanine green (ICG) clearance test. Although widely used, discrepancies have been reported for the ICG clearance test in relation with clinical outcome. Nuclear imaging studies have the advantage of providing simultaneous morphologic (visual) and physiologic (quantitative functional) information about the liver. In addition, regional (segmental) differentiation allows specific functional assessment of the future remnant liver. Technetium-99m ( $\left.{ }^{99 \mathrm{~m}} \mathrm{Tc}\right)$-galactosyl human serum albumin scintigraphy and ${ }^{99 m}$ Tc-mebrofenin hepatobiliary scintigraphy potentially identify patients at risk for post-resectional liver failure who might benefit from liver-augmenting techniques. As there is no one test that can measure all the components of liver function, liver functional reserve is estimated based on a combination of clinical parameters and quantitative liver function tests.

(C) 2017 S. Karger GmbH, Freiburg

\section{Introduction}

Liver-augmenting techniques such as portal vein embolization (PVE), 2-stage resection, and ALPPS (associating liver partition and portal vein ligation for staged hepatectomy) have pushed forward the frontiers of liver resection, resulting in an increase in the number of patients eligible for major liver resection. Judicious use of these modalities now offers patients with extensive tumor load, complex tumors, or highly compromised livers a chance for a curative resection. This aggressive surgical approach, however, comes with substantial morbidity and a mortality higher than accepted with standard resections.

The most dramatic complication after liver resection is the occurrence of posthepatectomy liver failure (PHLF) with reported mortality rates of up to $80 \%$ [1]. Management of PHLF is largely supportive, while these patients seldom are candidates for salvage (cadaveric) liver transplantation as the tumor burden underlying these extensive resections usually by far exceeds accepted criteria for liver replacement. Resection in these patients therefore remains a challenge and requires special skills and experience on the part of the hepatobiliary surgeons and all specialties involved in the preoperative assessment.

Many efforts have been directed toward predicting liver functional reserve preoperatively, taking into account the extent of resection considered necessary to remove all tumor and the quality of the liver parenchyma. We usually rely on computed tomography (CT) volumetric studies for the assessment of patients requiring major liver resection; however, while many of the patients referred for resection have undergone extensive chemotherapy with resulting steatotic or microvascular changes to their livers, the volume of the future remnant liver (FRL) may not correlate with the function of the FRL [2-4].

In this overview, we describe the current modalities available for the assessment of the FRL in patients scheduled for major hepatic resection. In patients with small-for-size FRL or insufficient FRL function, who are exposed to liver-augmenting techniques, we also

\section{KARGER}

() 2017 S. Karger GmbH, Freiburg

Fax +497614520714 
discuss the use of these functional tests to monitor the hypertrophy response of the FRL.

\section{Passive Liver Function Tests}

\section{Biochemical Parameters}

Passive liver function tests include biochemical parameters and clinical grading systems. The term 'liver function tests' to indicate a set of conventional plasma parameters is confusing because they do not represent the functional components of the liver except for clotting factors which are synthesized by the liver. The aminotransaminase enzymes, aspartate transferase (AST) and alanine transferase (ALT), are markers of liver damage and correlate with the extent of hepatocellular necrosis [5]. Although a persisting release of these enzymes will ultimately result in the loss of liver functional capacity, they are not parameters of function per se. Albumin and clotting factors, however, are exclusively synthesized by the liver and their plasma concentrations are therefore used as indirect indicators of liver function. The plasma bilirubin concentration provides indirect information on the uptake, conjugation, and excretion function of the liver. However, plasma bilirubin levels are also influenced by extrahepatic factors such as increased production of bilirubin or bile duct obstruction $[6,7]$.

Hyaluronic acid (HA), a more recent biochemical parameter, is a glycosaminoglycan which is produced by connective tissue cells and synovial cells and is exclusively taken up from the blood and metabolized by the sinusoidal endothelial cells of the liver. HA concentration in the blood may therefore be considered a functional test of the liver sinusoidal endothelial cells and as such of the whole liver. In a series of patients with primary and secondary liver tumors, we found a significant positive correlation between HA and almost every conventional liver parameter. A significant correlation was demonstrated between plasma concentrations of HA and the indocyanine green (ICG) retention test in which ICG retention of $15 \%$ corresponded with a HA level of $233 \mathrm{ng} / \mathrm{ml}$ (normal plasma HA values: $0-75 \mathrm{ng} / \mathrm{ml}$ ) (unpublished results). A similar correlation between plasma HA and ICG retention rate at $15 \mathrm{~min}$ (ICG-R15) was reported by Nanashima et al. [8].

\section{Clinical Grading Systems}

Clinical grading systems combine several biochemical parameters together with clinical symptoms of insufficient liver function. The Child-Pugh score is a widely used clinical scoring systems which includes total bilirubin plasma level, albumin plasma level, and prothrombin time (PT) together with the presence or absence of encephalopathy and ascites. The Child-Pugh scoring system is particularly useful in selecting patients with hepatocellular carcinoma (HCC) and cirrhosis for resection or transplantation, as most class Child B and class Child C patients are candidates for transplantation, leaving class Child A patients for resection. In patients with liver metastases who usually have normal liver parenchyma and who are all classified as class Child A, the question then arises of how to identify those at risk of PHLF [9]. In these patients, the Child-Pugh score has been shown to be quite variable and may be unreliable for predicting the outcome of liver resections, especially in those with non-cirrhotic livers [10].

The MELD (Model for End-Stage Liver Disease) score was originally developed to predict short-term survival in patients undergoing transcutaneous intrahepatic portosystemic shunt procedures and has been adopted to stratify patients with end-stage liver disease awaiting transplantation. The MELD score includes serum bilirubin and creatinine levels and INR (International Normalized Ratio). MELD scores, however, did not predict morbidity or mortality after elective liver resection [11].

\section{Dynamic Quantitative Liver Function Tests}

Biochemical liver function tests and clinical grading systems only provide indirect information about liver function. Therefore, especially in patients with non-cirrhotic livers, there is a need for objective tests to evaluate liver function in addition to clinical judgment. To this end, several dynamic quantitative tests of liver function have been devised [12]. As the various functional tests are based on different metabolic pathways, it is difficult to compare the value of each test in the context of risk assessment for liver resection. Several of these tests are discussed below.

\section{Indocyanine Green Clearance Test}

The ICG clearance test was initially devised for the measurement of blood flow, and later found clinical application in the assessment of liver function. It is now the most widely used quantitative liver function test. Although the test is routinely used in many Asian centers, its application has not been widely adopted in European centers. ICG is a tricarbocyanine dye that binds to albumin and is distributed uniformly in the blood within 2-3 min after intravenous injection. ICG is exclusively removed by the liver and excreted into the bile without intrahepatic conjugation [13]. Following administration, the blood level falls exponentially for about $20 \mathrm{~min}$, by which time approximately $97 \%$ of the dye is excreted. ICG clearance is determined by serum sampling or pulse dye densitometry using an optical sensor placed on the finger $[14,15]$.

The ICG clearance test was found to be the best discriminating preoperative test for evaluating hepatic functional reserve in patients with HCC [16]. However, there are some drawbacks that may lead to discrepancies in relation to histological findings and clinical outcome. Discrepancies have been reported between ICGR15 clearance rates and histological liver findings, and also clinical outcome [17]. Mortality has been noted in patients with normal ICG-R15, and survival in patients with poor outcome predicted on the basis of preoperative ICG-R15 [18]. One of the confounding factors in the measurement of ICG clearance is its flow dependency. Variation in hepatic blood flow such as that caused by intrahepatic shunting will influence the ICG clearance rate, rendering the test less predictive. Another point is that the ICG clearance test reflects the global liver function but does not take into account re- 
gional variations that may occur within the liver, obscuring a possible functional disadvantage of the segments to be preserved.

\section{Scintigraphic Liver Function Tests}

Technetium-99m( $\left.{ }^{99 m} \mathrm{Tc}\right)$-labeled diethylenetriaminepentaacetic acid (DTPA) galactosyl human serum albumin (GSA) scintigraphy and hepatobiliary scintigraphy (HBS) with ${ }^{99 \mathrm{~m}}$ Tc-labeled iminodiacetic acid (IDA) derivatives are the most common representatives of this group [19]. Although both methods are based on different principles, both provide quantitative and visual information on total and regional hepatic function. ${ }^{99 \mathrm{~m}} \mathrm{Tc}-\mathrm{GSA}$ scintigraphy is elaborated on and ${ }^{99} \mathrm{~m} \mathrm{Tc}-$ mebrofenin $\mathrm{HBS}$ is discussed in this section.

\section{${ }^{99 m}$ Tc-GSA Scintigraphy}

The asialoglycoprotein receptor is specific for asialoglycoproteins which are formed after the removal of sialic acid from endogenous glycoproteins by sialidases. Asialoglycoproteins bind to asialoglycoprotein receptors on the hepatocyte sinusoidal surface and are subsequently taken up by receptor-mediated endocytosis and delivered to lysosomes for degradation. Chronic liver disease is associated with a decrease in the amount of asialoglycoprotein receptors and an accumulation of plasma asialoglycoproteins [20]. The ${ }^{99 \mathrm{~m}}$ Tc-labeled asialoglycoprotein analog, ${ }^{99 \mathrm{~m}} \mathrm{Tc}-\mathrm{GSA}$, was clinically introduced as a new scintigraphy agent for imaging of the human hepatic receptor [21, 22]. ${ }^{99 \mathrm{~m} T c-G S A}$ is commercially available in an instant labelling kit in Japan.

The liver is the only uptake site for ${ }^{99 \mathrm{~m}} \mathrm{Tc}$-GSA which makes it an ideal agent for liver function assessment. Furthermore, the uptake of ${ }^{99 \mathrm{~m}} \mathrm{Tc}-\mathrm{GSA}$ is not affected by high bilirubin serum levels, making ${ }^{99 \mathrm{~m}} \mathrm{Tc}$-GSA scintigraphy applicable even in cholestatic patients. Despite years of experience in South-East Asia, this radiopharmaceutical agent has not been released for clinical use in most European countries.

\section{Hepatobiliary Scintigraphy Using IDA Derivatives}

${ }^{99 \mathrm{~m}} \mathrm{Tc}$-IDA agents are lidocain analogs that are taken up by the hepatocytes and are directly excreted into the bile canaliculi without undergoing any biotransformation. ${ }^{99 \mathrm{~m}} \mathrm{Tc}$-mebrofenin is the most hepatic-specific ${ }^{99} \mathrm{~m}$ Tc-IDA derivative [23]. Hepatic uptake of IDA agents via organic anion-transporting polypeptides (OATPs) can be influenced by high serum bilirubin levels as the same transporters are involved in the uptake of organic anions like bilirubin. Of all available IDA agents, ${ }^{99 \mathrm{~m}} \mathrm{Tc}$-mebrofenin shows the lowest displacement by bilirubin in the case of hyperbilirubinemia [24]. The uptake, excretion, and lack of hepatic biotransformation of the IDA agents are similar to ICG. These properties make IDA agents suitable for the imaging of the hepatobiliary system and for use in the diagnosis of different biliary diseases [25]. The high hepatic uptake, low displacement by bilirubin, and, furthermore, low urinary excretion make mebrofenin the most suitable IDA agent for hepatic function assessment. We have used HBS in our center since the year 2000 for risk assessment of patients due to undergo any resection comprising three or more Couinaud segments [26, 27].

Camera-based measurement of the relative hepatic uptake rate was developed by Ekman et al. [28]. After intravenous injection of freshly prepared ${ }^{99 \mathrm{~m}} \mathrm{Tc}$-mebrofenin, dynamic scintigraphy is performed with a dual-head gamma camera. Also here, the uptake of ${ }^{99} \mathrm{~m} \mathrm{Tc}$-mebrofenin is calculated by determining regions of interest (ROIs) around the heart, the liver, and the total field of view. Based on the ROIs, three time-activity curves can be generated. Using these parameters, it is possible to calculate the hepatic mebrofenin uptake ratio. Subsequently, a 3-dimensional single-photon emission computed tomography(SPECT)-CT is acquired during the time in which the tracer is accumulated in the liver. This provides quantitative information on the segmental distribution of function. A low-dose CT provides additional anatomical information to calculate the functional share of the FRL. As described by De Graaf et al. [29], the combination of dynamic HBS with SPECT-CT delivers visible and quantitative information regarding segmental liver function and therefore is an accurate measure of FRL function. The uptake ratio is divided by the body surface area and expressed as $\% / \mathrm{min} / \mathrm{m}^{2}$ in order to compensate for differences in individual metabolic requirements. Defining the FRL as ROI enables the estimation of specifically the function of the FRL [19].

The use of ${ }^{99 \mathrm{~m}} \mathrm{Tc}$-mebrofenin HBS for the preoperative assessment of liver function in patients undergoing liver surgery was first described by Erdogan et al. [30] and has since been adopted by several hepatobiliary units worldwide $[31,32]$. The hepatic uptake of mebrofenin can be calculated in the same way as for ICG. The mebrofenin uptake rate (MUR) strongly correlated with the ICG clearance test. Preoperatively measured FRL function with ${ }^{99 \mathrm{~m}} \mathrm{Tc}$ mebrofenin HBS proved to correlate with postoperative FRL function on postoperative day 1 [33]. Furthermore, in patients without parenchymal disease undergoing partial liver resection, preoperative measurement of ${ }^{99 \mathrm{~m}} \mathrm{Tc}$-mebrofenin uptake by the FRL was more accurate in predicting postoperative liver insufficiency and liver insufficiency-related mortality than preoperative measurement of FRL volume [34]. In surgical populations with and without compromised liver parenchyma, the MUR cut-off value was calculated at $2.7 / \mathrm{min} / \mathrm{m}^{2}$, making HBS more valuable in predicting postoperative liver failure compared to CT volumetry [26]. One single cut-off value for patients with compromised or non-compromised livers makes ${ }^{99 \mathrm{~m}} \mathrm{Tc}$-mebrofenin HBS an even more suitable liver function test in clinical practice, as the underlying liver disease is often unknown or poorly defined until resection has taken place. Liver biopsies are not routinely taken, as the distribution of compromised parenchyma in the liver is not homogeneous, leading to sampling errors, and because of the risk of biopsy-related complications [35]. This fact increases the value of ${ }^{99 \mathrm{~m}} \mathrm{Tc}$-mebrofenin HBS in daily practice. In cholestatic liver disease, however, such as in patients with perihilar cholangiocarcinoma, HBS readings may be affected depending on the completeness of the biliary drainage. Total or segmental MUR will be decreased parallel to and reflecting hyperbilirubinemia as a sign of secondary (obstructive) decreased liver function, which should be taken into consideration [36]. 


\section{New Modalities for Assessment of Liver Function}

\section{LiMAx Test $\left({ }^{13} \mathrm{C}\right.$-Methacetin Breath Test $)$}

There is a broad spectrum of ${ }^{13} \mathrm{C}$-breath tests available. The principle of the ${ }^{13} \mathrm{C}$-methacetin breath (LiMAx) test is based on the activity of the cytochrome P450 1A2 (CYP1A2) system, an enzyme system that is exclusively expressed in the liver [37]. The activity of this enzyme system proved to be reduced in patients with severe chronic liver disease regardless of cholestasis. CYP1A2 is distributed throughout the entire functional unit of the liver and is not affected by drugs or genetic variation. ${ }^{13} \mathrm{C}$-methacetin, the agent used to measure the activity of CYP1A2, is exclusively metabolized by the CYP1A2 system. ${ }^{13} \mathrm{C}$-methacetin is instantly metabolized to paracetamol and ultimately to ${ }^{13} \mathrm{CO}_{2}$, after which ${ }^{13} \mathrm{CO}_{2}$ is excreted through the lungs [38]. This causes alterations in the normal ${ }^{13} \mathrm{CO}_{2} /{ }^{12} \mathrm{CO}_{2}$ ratio in the breath that are analyzed by a modified, non-dispersive, isotope-selective, infrared, spectroscopy-based device. The expired air is collected using a specially designed face mask. In this manner, the ${ }^{13} \mathrm{C}$-methacetin breath test provides quantitative information on hepatic function.

The LiMAx test is a non-invasive and easy to perform test, which makes it an attractive option in clinical practice [39]. The cut-off value of normal LiMAx readout is set at $311-575 \mu \mathrm{g} / \mathrm{kg} / \mathrm{h}$. While LiMAx assesses total liver functional capacity, the test can be used to measure FRL function by combining it with CT-volumetric analysis of FRL. The authors assume that the percentage of liver function attributed to the FRL equals the percentage of FRL volume; however, this method does not take into account regional differences in liver function. On the other hand, preoperative FRL LiMAx values correlated with the LiMAx values measured on the first postoperative day. The LiMAx value on postoperative day 1 has also been described as a predictor of PHLF and liver failure-related mortality. The same research group proposed a decision-tree based on the LiMAx results, which is supposed to help surgeons to decide between resection and alternative or additional therapies like PVE, neoadjuvant treatment, and palliative therapy [40]. The value of this algorithm and the proposed cut-off values await further clinical assessment in a prospective setting.

The major limitation of the ${ }^{13} \mathrm{C}$-breath tests is the assumption that the contribution of the FRL to total liver function is equal to the proportion of FRL to total liver volume. Inhomogeneous distribution of liver function throughout the liver has been demonstrated using scintigraphic methods as well as with magnetic resonance imaging (MRI) [41, 42]. Another difficulty in the application of the LiMAx test is that the test results are potentially affected by several factors such as hemodialysis, smoking, nutrition, and visceral hemodynamics [40]. Also, members of the CYP1A family are considerably downregulated in HCC, rendering the test less universal in its use for the entire population of patients requiring liver resection [43].

Assessment of Liver Function Using Magnetic Resonance Imaging MRI with gadolinium ethoxybenzyl (Gd-EOB)-DTPA is well established as a liver imaging technique. MRI provides accurate anatomical information and has recently also been introduced as a potential technique for the preoperative assessment of liver function [44-46]. Contrast-enhanced (CE) MRI with Gd-based contrast agents allows a more accurate depiction of benign or malign liver lesions than CT [47].

The concept of using CE-MRI with Gd-EOB-DTPA in the evaluation of liver function was first introduced in 1993 [48]. Subsequently, several studies were published showing a correlation between MRI with Gd-EOB-DTPA and liver function in animal models [49-51]. Recently, data on the assessment of liver function using MRI with Gd-EOB-DTPA in humans were published, all of them confirming the possibility of liver function assessment using MRI [52-54].

Functional imaging with Gd-EOB-DTPA-enhanced MRI has also been used for the assessment of total and regional liver function [55]. Given that MRI now allows the segmental assessment of steatosis and can be used to assess fibrosis, this would make it a potential '1-stop-shop' modality for both liver anatomy and function $[56,57]$. However, the use of MRI with Gd-EOB-DTPA for the assessment of liver function is still under investigation. Technical difficulties have to be resolved concerning the molar amount of tracer needed for visualization on MRI, as well as issues regarding the absolute quantification of function.

\section{Assessment of Increase in FRL Function after PVE}

Postoperative regeneration of the remnant liver is usually evaluated by CT volumetry. Failure of the non-embolized liver segments to hypertrophy is considered a significant sign of a deficient functional reserve of the liver, increasing the risk of liver failure associated with liver resection. As postresectional regeneration depends on the same mechanisms, insufficient volume increase of the FRL after PVE is a strong predictor of postoperative liver failure $[58,59]$.

A decreased ICG clearance rate has been used as a guide when considering preoperative PVE in patients requiring major liver resection in order to augment FRL function. Conceivably, as ICG clearance measures global liver function, an increase in function of the non-embolized lobe is accompanied by decreased function of the embolized atrophied lobe, resulting in no net effect on total liver function. The ICG clearance test is therefore not able to specifically measure the increase in FRL function after PVE.

Scintigraphic techniques are gaining applicability in patients undergoing PVE. Multiple studies evaluated the increase in FRL function after PVE using ${ }^{99 \mathrm{~m}} \mathrm{Tc}$-GSA scintigraphy [60-62]. The increase in ${ }^{99 \mathrm{~m}} \mathrm{Tc}$-GSA uptake in the FRL was found to be more extensive than the increase in FRL volume. Using ${ }^{99 \mathrm{~m}} \mathrm{Tc}$-mebrofenin HBS, it was likewise concluded that the increase in FRL function exceeded the increase in FRL volume $[29,63]$. These findings suggest that the time interval between PVE and liver resection may be shorter than determined on the basis of volumetric parameters. Another possible application of HBS in this group of patients is the selection of candidates for PVE or ALPPS. A recent report showed that FRL function assessed with HBS can be used as a predictor of 
insufficient functional hypertrophy after PVE, especially in nonchemotherapy patients. These patients are potential candidates for upfront ALPPS [64].

The LiMAx test has recently been explored in patients undergoing PVE [65]. In this study, LiMAx was used to visualize the changes in FRL function in the time between PVE and major liver resection, showing an increase in FRL function after PVE. Furthermore, the authors found that FRL function post-resection was lower than the preoperatively calculated function, which they explain by loss of function due to intraoperative injury. The authors recommend that an overestimation margin of the FRL is needed preoperatively in order to compensate for this loss, which renders the test less accurate in this particular setting.

\section{Assessment of Increase in FRL Function after Stage 1 in ALPPS}

In ALPPS, the timing of stage 2, i.e. resection of the deportalized liver lobe, is a much debated issue. The usual imaging modality to assess hypertrophy of the FRL after stage 1 is CT volumetry. As volumetric studies do not consistently correlate with liver function, interstage CT volumetry may be unreliable as a predictor of FRL function in the setting of ALPPS. This notion is corroborated by reports that liver failure after ALPPS stage 2 occurred with an incidence of $30 \%$ [66]. Assessment of FRL function therefore seems of great importance for the timing of stage 2. A possible explanation for the discrepancy between volume and function after stage 1 is that hepatocytes after cell division need time to mature before full function can be exerted and that 7-14 days is simply too short a period for function to develop [67].

As discussed above, the ICG clearance test depends on overall liver blood flow and is therefore less applicable during ALPPS because of the division of the portal vein and redistribution of portal and hepatic arterial flow. Furthermore, the ICG clearance test is a global liver function test and does not provide information on specifically segmental function, i.e. the FRL.

Scintigraphic liver function tests such as HBS using ${ }^{99 \mathrm{~m}} \mathrm{Tc}-\mathrm{la}-$ beled IDA derivatives have the potential to provide quantitative and visual information on total and regional hepatic function. Sequential measurement of FRL function using HBS therefore seems an advantage in ALPPS. By monitoring inter-stage liver function using HBS, the second stage, i.e. resection of the deportalized lobe, can be safely undertaken [68]. Recently, the use of HBS for the timing of stage 2 in ALPPS was compared with CT volumetry in 60 patients completing ALPPS in 6 European centers. The results showed that volumetry overestimated the liver function [69].

\section{Discussion}

The function of the liver is complex, representing a spectrum of functional processes that can grossly be divided into four categories: uptake, synthesis, biotransformation, and excretion [6]. There is no one functional test that represents the multiple components of liver function, and along the same lines, there is no single test that can accurately predict operative risk in patients considered for major liver resection. We still rely mostly on the combination of clinical parameters and volumetric studies to estimate liver functional reserve and to decide whether we can perform a safe resection. Several techniques are now available for the assessment of liver function of which passive liver tests and dynamic quantitative liver function tests have been employed most frequently. It is time for a change in paradigm, moving from volumetrics to functionometrics in the assessment of surgical risk in patients requiring major liver resection.

Several clinical and quantitative liver function tests have been described in the literature. The Child-Pugh classification, based on clinical symptoms of insufficient liver function (ascites/encephalopathy), and laboratory analysis of parameters of liver function (albumin, bilirubin, and PT) can be used to identify high-risk patients, i.e., class Child B and class Child C. The challenge is, however, to select among class Child A patients those who are goodrisk and those who are poor-risk patients [9]. In these patients, reliable quantitative liver function tests are crucial.

Specific liver function tests such as the ICG clearance rate can complement the clinical Child-Pugh classification. The ICG clearance rate has been reported not to unequivocally correlate with morbidity and mortality after partial liver resection. The reported studies, however, vary with respect to the extent of the liver resections performed and the type of patients included (i.e. patients with HCC and cirrhosis vs. patients with normal liver parenchyma). Another drawback of the ICG clearance test is that only global liver function is measured whereas one would be particularly interested in the functional capacity of the part of the liver to be preserved during liver resection. If plasma ICG clearance is multiplied by the proportion of FRL volume, the plasma ICG clearance of specifically the FRL can be calculated. This has been shown to be predictive of PHLF with a cut-off value of 0.05 [70]. This is, however, only applicable in patients with homogenous liver function, making it less feasible in cases of PVE or chronic liver disease.

Nuclear imaging studies have been introduced for the quantitative testing of liver function. Potential advantages of these techniques are the possibility to provide simultaneous morphologic (visual) and physiologic (functional) information of the liver, especially when SPECT-CT cameras are used. Functional capacity may vary within the liver, and with nuclear imaging studies regional differences in hepatic function are detected. SPECT allows measurement of function of specifically the FRL. In addition, when using radiopharmaceutical agents that are excreted into the bile, two dynamic phases can be examined, i.e. hepatic uptake of the agent and secretion into the biliary system. Using ${ }^{99 \mathrm{~m}} \mathrm{Tc}$-mebrofenin scintigraphy, definition of a single cut-off value (i.e., $2.7 / \mathrm{min} / \mathrm{m}^{2}$ ) allows the use of a '1-size-fits-all' test to assess non-cholestatic patients irrespective of the quality of liver parenchyma.

Both ${ }^{99 \mathrm{~m}} \mathrm{Tc}$-GSA and ${ }^{99 \mathrm{~m}} \mathrm{Tc}$-mebrofenin scintigraphy have the potential to preoperatively select patients with a high risk of developing postoperative liver insufficiency who might therefore benefit 
from PVE. After PVE, hypertrophy of the non-embolized liver segments is commonly monitored by CT volumetry, showing only the morphologic increase in the FRL. However, little is known about the increase in FRL function after PVE with only few available methods to measure FRL function. Although the ICG clearance test is able to measure liver function, this test only quantifies overall liver function and is therefore less suitable for the assessment of post-PVE increase in FRL function. Nuclear imaging studies, however, provide information on augmented function of the FRL. A discrepancy between volumetric hypertrophy and functional increase has been described in several studies, indicating that functional gain of the FRL is more rapid and of greater magnitude than the volumetric increase. Since after PVE the functional advantage of the remnant liver exceeds the volume increase of the residual liver, the recommended waiting time following PVE may be shorter than suggested by volumetric studies [63]. The reverse has been observed in patients undergoing ALPPS in whom in the short time after stage 1 volume exceeds function [69].

There is no one test that can accurately predict the surgical risk in patients considered for major liver resection. Clearly, scoring methods need to be developed in which clinical parameters, CT volumetric criteria, and the results of dynamic quantitative liver function tests guide our decision-making in patients requiring major liver resection. Objective functional criteria are necessary to define patients at increased risk and select those who might benefit from preoperative liver-augmenting techniques.

\section{Disclosure Statement}

The authors have no conflict of interest to disclose.

\section{References}

1 Van den Broek MA, Olde Damink SW, Dejong CH, et al: Liver failure after partial hepatic resection: definition, pathophysiology, risk factors and treatment. Liver Int 2008;28:767-780.

2 Shoup M, Gonen M, D'Angelica M, et al: Volumetric analysis predicts hepatic dysfunction in patients undergoing major liver resection. J Gastrointest Surg 2003; 7:325-330.

3 Vauthey JN, Chaoui A, Do KA, et al: Standardized measurement of the future liver remnant prior to extended liver resection: methodology and clinical associations. Surgery 2000;127:512-519.

4 Martel G, Cieslak KP, Huang R, et al: Comparison of techniques for volumetric analysis of the future liver remnant: implications for major hepatic resections. HPB (Oxford) 2015;17:1051-1057.

5 Field KM, Dow C, Michael M: Part I: Liver function in oncology: biochemistry and beyond. Lancet Oncol 2008;9:1092-1101

6 Hoekstra LT, de Graaf W, Nibourg GA, et al: Physiological and biochemical basis of clinical liver function tests: a review. Ann Surg 2013;257:27-36.

7 Roberts KJ, Bharathy KG, Lodge JP: Kinetics of liver function tests after a hepatectomy for colorectal liver metastases predict post-operative liver failure as defined by the International Study Group for Liver Surgery. HPB (Oxford) 2013;15:345-351.

8 Nanashima A, Yamaguchi H, Shibasaki S, et al: Measurement of serum hyaluronic acid level during the perioperative period of liver resection for evaluation of functional liver reserve. J Gastroenterol Hepatol 2001; $16: 1158-1163$

9 Schneider PD: Preoperative assessment of liver func tion. Surg Clin North Am 2004;84:355-373.

10 Nagashima I, Takada T, Okinaga K, Nagawa H: A scoring system for the assessment of the risk of mortality after partial hepatectomy in patients with chronic liver dysfunction. J Hepatobiliary Pancreat Surg 2005; 12:44-48.

11 Schroeder RA, Marroquin CE, Bute BP, et al: Predictive indices of morbidity and mortality after liver resection. Ann Surg 2006;243:373-379.

12 Cieslak KP, Runge JH, Heger M, et al: New perspectives in the assessment of future remnant liver. Dig Surg 2014;31:255-268.
13 Paumgartner G: The handling of indocyanine green by the liver. Schweiz Med Wochenschr 1975;105(suppl): $1-30$

14 Okochi O, Kaneko T, Sugimoto H, et al: ICG pulse spectrophotometry for perioperative liver function in hepatectomy. J Surg Res 2002;103:109-113.

15 Akita H, Sasaki Y, Yamada T, et al: Real-time intraoperative assessment of residual liver functional reserve using pulse dye densitometry. World J Surg 2008;32: 2668-2674.

16 Lau H, Man K, Fan ST, et al: Evaluation of preoperative hepatic function in patients with hepatocellular carcinoma undergoing hepatectomy. Br J Surg 1997; 84:1255-1259.

17 Lam CM, Fan ST, Lo CM, Wong J: Major hepatectomy for hepatocellular carcinoma in patients with an unsatisfactory indocyanine green clearance test. $\mathrm{Br} \mathrm{J}$ Surg 1999;86:1012-1017.

18 Fan ST, Lai EC, Lo CM, et al: Hospital mortality of major hepatectomy for hepatocellular carcinoma associated with cirrhosis. Arch Surg 1995;130:198-203.

19 De Graaf W, Bennink RJ, Veteläinen R, van Gulik TM: Nuclear imaging techniques for the assessment of hepatic function in liver surgery and transplantation. J Nucl Med 2010;51:742-752.

20 Garcea G, Ong SL, Maddern GJ: Predicting liver failure following major hepatectomy. Dig Liver Dis 2009;41: 798-806.

21 Suzuki Y, Kohno Y, Takeda Y, Hiraki Y: Evaluation of liver function parameters by Tc-99m-GSA using multivariate analysis: a study of 47 clinical cases. Acta Med Okayama 1999;53:225-232.

22 Kokudo N, Vera DR, Makuuchi M: Clinical application of TcGSA. Nucl Med Biol 2003;30:845-849.

23 De Graaf W, Häusler S, Heger M, et al: Transporters involved in the hepatic uptake of ${ }^{99 \mathrm{~m}} \mathrm{Tc}$-mebrofenin and indocyanine green. J Hepatol 2011;54:738-745.

24 Ghibellini G, Leslie EM, Pollack GM, Brouwer KL: Use of tc- $99 \mathrm{~m}$ mebrofenin as a clinical probe to assess altered hepatobiliary transport: integration of in vitro, pharmacokinetic modeling, and simulation studies. Pharm Res 2008;25:1851-1860.

25 Trauner M, Meier PJ, Boyer JL: Molecular pathogenesis of cholestasis. N Engl J Med 1998;339:1217-1227.
26 De Graaf W, van Lienden KP, Dinant S, et al: Assessment of future remnant liver function using hepatobiliary scintigraphy in patients undergoing major liver resection. J Gastrointest Surg 2010;14:369-378.

27 Cieslak KP, Bennink RJ, de Graaf W, et al: Measurement of liver function using hepatobiliary scintigraphy improves risk assessment in patients undergoing major liver resection. HPB (Oxford) 2016;18:773-780.

28 Ekman M, Fjälling M, Friman S, et al: Liver uptake function measured by IODIDA clearance rate in liver transplant patients and healthy volunteers. Nucl Med Commun 1996;17:235-242.

29 De Graaf W, van Lienden KP, van Gulik TM, Bennink RJ: ${ }^{99 m} \mathrm{Tc}-$ mebrofenin hepatobiliary scintigraphy with SPECT for the assessment of hepatic function and liver functional volume before partial hepatectomy. J Nucl Med 2010;51:229-236.

30 Erdogan D, Heijnen BH, Bennink RJ, et al: Preoperative assessment of liver function: a comparison of $99 \mathrm{mTc}-\mathrm{mebrofenin}$ scintigraphy with indocyanine green clearance test. Liver Int 2004;24:117-123.

31 Serenari M, Collaud C, Alvarez FA, et al: Interstage assessment of remnant liver function in ALPPS using hepatobiliary scintigraphy: prediction of posthepatectomy liver failure and introduction of the HIBA index. Ann Surg 2017;DOI: 10.1097/SLA.0000000000002150.

32 Chapelle T, Op De Beeck B, Huyghe I, et al: Future remnant liver function estimated by combining liver volumetry on magnetic resonance imaging with total liver function on ${ }^{99 \mathrm{~m}} \mathrm{Tc}$-mebrofenin hepatobiliary scintigraphy: can this tool predict post-hepatectomy liver failure? HPB (Oxford) 2016;18:494-503.

33 Bennink RJ, Dinant S, Erdogan D, et al: Preoperative assessment of postoperative remnant liver function using hepatobiliary scintigraphy. J Nucl Med 2004;45: 965-971.

34 Dinant S, de Graaf W, Verwer BJ, et al: Risk assessment of posthepatectomy liver failure using hepatobiliary scintigraphy and CT volumetry. J Nucl Med 2007; 48:685-692.

35 Ratziu V, Charlotte F, Heurtier A, et al: Sampling variability of liver biopsy in nonalcoholic fatty liver disease. Gastroenterology 2005;128:1898-1906. 
36 Olthof PB, Coelen RJS, Bennink RJ, et al: 99mTc-mebrofenin hepatobiliary scintigraphy predicts liver failure following major liver resection for perihilar cholangiocarcinoma. HPB (Oxford) 2017;19:850-858.

37 Guengerich FP, Krauser JA, Johnson WW: Rate-limiting steps in oxidations catalyzed by rabbit cytochrome P450 1A2. Biochemistry 2004;43:10775-10788.

38 Schoeller DA, Schneider JF, Solomons NW, et al: Clin ical diagnosis with the stable isotope $13 \mathrm{C}$ in $\mathrm{CO}_{2}$ breath tests: methodology and fundamental considerations. J Lab Clin Med 1977;90:412-421.

39 Stockmann M, Lock JF, Riecke B, et al: Prediction of postoperative outcome after hepatectomy with a new bedside test for maximal liver function capacity. Ann Surg 2009;250:119-125.

40 Stockmann M, Lock JF, Malinowski M, et al: The LiMAx test: a new liver function test for predicting postoperative outcome in liver surgery. HPB (Oxford) 2010;12:139-146.

41 Sumiyoshi T, Shima Y, Tokorodani R, et al: CT/ 99mTc-GSA SPECT fusion images demonstrate functional differences between the liver lobes. World J Gastroenterol 2013;19:3217-3225.

42 Nilsson H, Karlgren S, Blomqvist L, Jonas E: The inhomogeneous distribution of liver function: possible impact on the prediction of post-operative remnant liver function. HPB (Oxford) 2015; 17:272-277.

43 McKinnon RA, Hall PD, Quattrochi LC, et al: Localization of CYP1A1 and CYP1A2 messenger RNA in normal human liver and in hepatocellular carcinoma by in situ hybridization. Hepatology 1991;14:848-856.

44 Nilsson H, Nordell A, Vargas R, et al: Assessment of hepatic extraction fraction and input relative blood flow using dynamic hepatocyte-specific contrast-enhanced MRI. J Magn Reson Imaging 2009;29:1323-1331.

45 Sourbron S, Sommer WH, Reiser MF, Zech CJ: Combined quantification of liver perfusion and function with dynamic gadoxetic acid-enhanced MR imaging. Radiology 2012;263:874-883.

46 Saito K, Ledsam J, Sourbron S, et al: Measuring hepatic functional reserve using low temporal resolution GdEOB-DTPA dynamic contrast-enhanced MRI: a preliminary study comparing galactosyl human serum albumin scintigraphy with indocyanine green retention. Eur Radiol 2014;24:112-119.

47 Niekel MC, Bipat S, Stoker J: Diagnostic imaging of colorectal liver metastases with CT, MR imaging, FDG PET, and/or FDG PET/CT: a meta-analysis of prospective studies including patients who have not previously undergone treatment. Radiology 2010;257:674-684.
48 Schuhmann-Giampieri G: Liver contrast media for magnetic resonance imaging. Interrelations between pharmacokinetics and imaging. Invest Radiol 1993;28: 753-761.

49 Ryeom HK, Kim SH, Kim JY, et al: Quantitative evaluation of liver function with MRI using Gd-EOB-DTPA. Korean J Radiol 2004;5:231-239.

50 Tsuda N, Okada M, Murakami T: New proposal for the staging of nonalcoholic steatohepatitis: evaluation of liver fibrosis on Gd-EOB-DTPA-enhanced MRI. Eur J Radiol 2010;73:137-142.

51 Tsuda N, Okada M, Murakami T: Potential of gadolinium-ethoxybenzyl-diethylenetriamine pentaacetic acid (Gd-EOB-DTPA) for differential diagnosis of nonalcoholic steatohepatitis and fatty liver in rats using magnetic resonance imaging. Invest Radiol 2007;42:242247.

52 Nilsson H, Blomqvist L, Douglas L, et al: Gd-EOBDTPA-enhanced MRI for the assessment of liver function and volume in liver cirrhosis. Br J Radiol 2013;86: 20120653.

53 Motosugi U, Ichikawa T, Sou H, et al: Liver parenchymal enhancement of hepatocyte-phase images in GdEOB-DTPA-enhanced MR imaging: which biological markers of the liver function affect the enhancement? J Magn Reson Imaging 2009;30:1042-1046.

54 Tajima T, Takao H, Akai H, et al: Relationship between liver function and liver signal intensity in hepatobiliary phase of gadolinium ethoxybenzyl diethylenetriamine pentaacetic acid-enhanced magnetic resonance imaging. J Comput Assist Tomogr 2010;34:362-366.

55 Nilsson H, Blomqvist L, Douglas L, et al: Assessment of liver function in primary biliary cirrhosis using GdEOB-DTPA-enhanced liver MRI. HPB (Oxford) 2010; 12:567-576.

56 Van Werven JR, Marsman HA, Nederveen AJ, et al: Assessment of hepatic steatosis in patients undergoing liver resection: comparison of US, CT, T1-weighted dual-echo MR imaging, and point-resolved $1 \mathrm{H}$ MR spectroscopy. Radiology 2010;256:159-168.

57 Venkatesh SK, Yin M, Ehman RL: Magnetic resonance elastography of liver: technique, analysis, and clinical applications. J Magn Reson Imaging 2013;37:544-555.

58 Ribero D, Abdalla EK, Madoff DC, et al: Portal vein embolization before major hepatectomy and its effects on regeneration, resectability and outcome. Br J Surg 2007;94:1386-1394.

59 Van Gulik TM, van den Esschert JW, de Graaf W, et al: Controversies in the use of portal vein embolization. Dig Surg 2008;25:436-444.
60 Hirai I, Kimura W, Fuse A, et al: Evaluation of preoperative portal embolization for safe hepatectomy, with special reference to assessment of nonembolized lobe function with $99 \mathrm{mTc}$-GSA SPECT scintigraphy. Surgery 2003;133:495-506.

61 Nishiyama Y, Yamamoto Y, Hino I, et al: ${ }^{99 \mathrm{~m}} \mathrm{Tc}$ galactosyl human serum albumin liver dynamic SPET for pre-operative assessment of hepatectomy in relation to percutaneous transhepatic portal embolization. Nucl Med Commun 2003;24:809-817.

62 Nanashima A, Yamaguchi H, Shibasaki S, et al: Relationship between CT volumetry and functional liver volume using technetium- $99 \mathrm{~m}$ galactosyl serum albumin scintigraphy in patients undergoing preoperative portal vein embolization before major hepatectomy: a preliminary study. Dig Dis Sci 2006;51:1190-1195.

63 De Graaf W, van Lienden KP, van den Esschert JW, et al: Increase in future remnant liver function after preoperative portal vein embolization. Br J Surg 2011;98: 825-834.

64 Cieslak KP, Huisman F, Bais T, et al: Future remnant liver function as predictive factor for the hypertrophy response after portal vein embolization. Surgery 2017; 162:37-47.

65 Malinowski M, Lock JF, Seehofer D, et al: Preliminary study on liver function changes after trisectionectomy with versus without prior portal vein embolization. Surg Today 2016;46:1053-1061.

66 Schadde E, Raptis DA, Schnitzbauer AA, et al: Prediction of mortality after ALPPS stage-1: an analysis of 320 patients from the International ALPPS Registry. Ann Surg 2015;262:780-785; discussion 785-786.

67 Matsuo K, Murakami T, Kawaguchi D, et al: Histologic features after surgery associating liver partition and portal vein ligation for staged hepatectomy versus those after hepatectomy with portal vein embolization. Surgery 2016;159:1289-1298.

68 Cieslak KP, Olthof PB, van Lienden KP, et al: Assessment of liver function using ${ }^{99 \mathrm{~m}} \mathrm{Tc}$-mebrofenin hepatobiliary scintigraphy in ALPPS (associating liver partition and portal vein ligation for staged hepatectomy). Case Rep Gastroenterol 2015;9:353-360.

69 Olthof PB, Tomassini F, Huespe PE, et al: Hepatobiliary scintigraphy to evaluate liver function in associating liver partition and portal vein ligation for staged hepatectomy: liver volume overestimates liver function. Surgery 2017;162:775-783.

70 Yokoyama Y, Nishio H, Ebata T, et al: Value of indocyanine green clearance of the future liver remnant in predicting outcome after resection for biliary cancer. Br J Surg 2010;97:1260-1268. 